\title{
Bile reflux gastritis and Barrett's oesophagus: further evidence of a role for duodenogastro-oesophageal reflux?
}

\author{
M F Dixon, P M Neville, N P Mapstone, P Moayyedi, A T R Axon
}

\begin{abstract}
Background-There is increasing evidence that reflux of bile plays a part in the pathogenesis of Barrett's oesophagus. Bile injury to the gastric mucosa results in a "chemical" gastritis in which oedema and intestinal metaplasia are prominent.

Aim-To determine if patients with Barrett's oesophagus have more bile related changes in antral mucosa than patients with uncomplicated gastro-oesophageal reflux disease (GORD) or non-ulcer dyspepsia (NUD).
\end{abstract}

Patients and methods-Patients were identified by a retrospective search of pathology records and those with a clinically confirmed diagnosis of either Barrett's oesophagus or reflux oesophagitis who had oesophageal and gastric biopsies taken at the same endoscopy and had no evidence of Helicobacter pylori infection entered the study. Control biopsies were taken from $H$ pylori negative NUD patients. Antral biopsies were examined "blind" to clinical group and graded for a series of histological features from which the "reflux gastritis score" (RGS) and "bile reflux index" (BRI) could be calculated. The reproducibility of these histological scores was tested by a second pathologist.

Results-There were 100 patients with Barrett's, 61 with GORD, and 50 with NUD. The RGSs did not differ between groups. BRI values in the Barrett's group were significantly higher than those in GORD subjects $(p=0.014)$ which in turn were higher than those in NUD patients $(p=0.037)$. Similarly, the frequency of high BRI values $(>14)$ was significantly greater in the Barrett's group $(29 / 100 ; 29 \%)$ than in the GORD $(9 / 61 ; 14.8 \%)$ or NUD $(4 / 50$; $8 \%)$ group. However, agreement on BRI values was "poor", indicating limited applicability of this approach.

Conclusion-Patients with Barrett's oesophagus have more evidence of bile related gastritis than subjects with uncomplicated GORD or NUD. The presence of bile in the refluxate could be a factor in both the development of "specialised" intestinal metaplasia and malignancy in the oesophagus. (Gut 2001;49:359-363)

Keywords: Barrett's oesophagus; reflux gastritis; bile; duodenogastro-oesophageal reflux; intestinal metaplasia
Barrett's oesophagus is widely considered to be a consequence of longstanding acid induced injury commencing as an erosive oesophagitis and progressing over years to columnar and intestinal metaplasia of the squamous epithelium. The process of metaplasia represents a non-neoplastic change in cellular phenotype which is thought to be a response to a sustained adverse environment. ${ }^{1}$ The change may be a consequence of somatic mutation in the epithelial stem cells or an epigenetic event whereby divergent differentiation in progeny cells produces the altered phenotype. ${ }^{2}$ Whatever the precise mechanism, the resulting cell lineage has a survival advantage over "native" epithelium so that selection pressures promote the emergence and dominance of the metaplastic population. Examples in the gastrointestinal tract include gastric metaplasia in the duodenum in response to acid injury, ${ }^{3}$ intestinal metaplasia in the stomach in longstanding Helicobacter pylori infection, ${ }^{45}$ and colonic metaplasia in ileal pouches in response to acquisition of a faecal-type flora. ${ }^{6}$ Following this line of reasoning, the appearance of gastric metaplasia in the lower oesophagus - that is, proximal extension of cardia-type mucosa-is a logical adaptive response to sustained acid reflux. However, acid injury alone would not be expected to induce intestinal metaplasia, the defining feature of Barrett's oesophagus.

Recent evidence points to a role for bile reflux in Barrett's oesophagus. ${ }^{7}$ The presence of bile in the oesophagus obviously necessitates duodenogastro-oesophageal reflux (DGOR) with passage of initially alkaline duodenal contents through the stomach. ${ }^{8}$ We have previously shown that bile reflux produces consistent histological changes in the gastric mucosa, ${ }^{9}$ and that an index based on these features was predictive of elevated bile acids in the refluxate. ${ }^{10}$ More interestingly, we found a stronger association between raised intragastric bile acid levels and incomplete (type III) intestinal metaplasia than with the complete form (type I) ${ }^{10}$ Incomplete intestinal metaplasia is the phenotype found in Barrett's oesophagus. These findings suggested to us that bile, as part of DGOR, could have a role in producing intestinal metaplasia in the oesophagus. If this is so, antral histology is more likely to reflect bile related changes in patients with Barrett's oesophagus

Abbreviations used in this paper: DGOR, duodenogastro-oesophageal reflux; GORD, gastro-oesophageal reflux disease; NUD, non-ulcer dyspepsia; BRI, bile reflux index; RGS, reflux gastritis score; NSAIDs, non-steroidal anti-inflammatory drugs. 


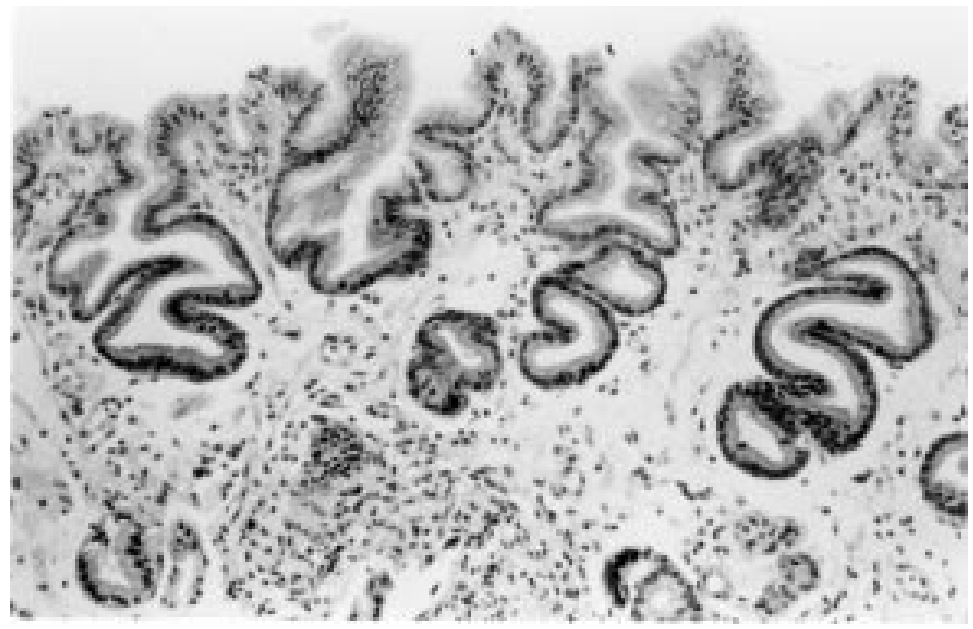

Figure 1 Antral biopsy from a patient with Barrett's oesophagus. This was graded as: foveolar hyperplasia =2; congestion $=0$; acute inflammation $=0$; chronic inflammation $=1$; oedema $=2$; intestinal metaplasia $=0$. These grades give a reflux gastritis score of 9 and a bile reflux index of 18 . An index $>14$ is taken as a strong predictor of a raised gastric juice bile acid content.

than in control subjects. In order to explore this hypothesis, we undertook a retrospective examination of gastric histology in patients with established Barrett's oesophagus, in patients with gastro-oesophageal reflux disease (GORD) without Barrett's, and in control subjects with no symptoms of GORD or endoscopic evidence of erosive oesophagitis or Barrett's metaplasia.

\section{Methods and patients}

REFLUX GASTRITIS SCORE AND BILE REFLUX INDEX

In our original study on reflux gastritis, we described a distinctive histological picture comprising foveolar hyperplasia $(\mathrm{FH})$, lamina propria vasodilatation and congestion (VC), oedema (Oed), and a paucity of acute (AI) and chronic (CI) inflammatory cells. ${ }^{9}$ Using grades (0-3) for each of these features a "reflux gastritis score" (RGS) was devised which comprised $(\mathrm{FH}+\mathrm{VC}+\mathrm{Oed})-(\mathrm{AI}+\mathrm{CI})+6$; the addition of " 6 " is to compensate for the inflammatory cell grades so that the overall score reflects their paucity. Thus cases scoring 3 for both AI and CI will effectively have nothing added whereas biopsies devoid of inflammatory cells have 6 points added. The RGS ranges from 0 to 15 and in the past we have taken scores $>10$ to be indicative of "reflux gastritis". A higher frequency of such scores was found in subjects with increased intragastric bile acids. ${ }^{9}$ However, we subsequently appreciated that many subjects with a high RGS did not have evidence of bile reflux; a proportion were taking non-steroidal anti-inflammatory drugs (NSAIDs), some had high alcohol consumption, and in many instances no cause was identified. ${ }^{11}$ We therefore sought a more accurate predictor of bile reflux.

The bile reflux index (BRI) was derived by stepwise logistic regression analysis of the histological grades used in the RGS together with intestinal metaplasia (IM) and $H$ pylori colonisation $(H p)$ found in gastric biopsies from 350 subjects in whom gastric juice bile acid levels had been measured. ${ }^{10}$ Following analysis, an index comprising $(7 \times$ Oed $)+(3 \times \mathrm{IM})+(4 \times \mathrm{CI})-(6 \times H p)$ gave the best prediction of a raised gastric juice bile acid concentration. An index above 14 had a sensitivity of $70 \%$ and a specificity of $85 \%$ for a bile acid level $>1.00 \mathrm{mmol} / 1$ (the upper limit of "physiological" reflux). As we wished to investigate only $H$ pylori negative subjects in this study (see below), we used the original data to examine the predictive value of the BRI in negative cases. In the $128 \mathrm{H}$ pylori negative cases, a BRI $>14$ had a sensitivity of $79.2 \%$ and a specificity of $82.7 \%$ for a bile acid concentration $>1 \mathrm{mmol} / \mathrm{l}$. In so far as $H$ pylori colonisation has a negative effect on BRI, the values obtained in a particular clinical group will be affected by the prevalence of infection in that group. Additionally, the degree of $\mathrm{H}$ pylori colonisation and chronic inflammation in the antrum in positive cases will be affected by concurrent treatment with proton pump inhibitors which will differ between groups. ${ }^{12}$ Finally, recognition of lamina propria oedema and other histological features of reflux gastritis are more difficult in $H$ pylori positive cases. ${ }^{13}$ For these reasons, we have chosen to compare only $H$ pylori negative subjects.

\section{PATIENT GROUPS}

A computer search of pathology records from August 1998 to July 1999 revealed 396 patients whose oesophageal biopsies carried a diagnosis of reflux (erosive) oesophagitis and 474 patients with Barrett's oesophagus. Of these, a total of 227 had gastric biopsies taken at the same endoscopy. The clinical details of each patient were checked and any not fulfilling the correct clinical and/or endoscopic diagnosis were eliminated, as were subjects with previous gastric surgery. At the same time, on the basis of clinical, endoscopic, and histological findings, patients were allocated to either mild/ moderate or severe GORD, and either long or short $(<3 \mathrm{~cm})$ segment Barrett's oesophagus. There were 79 patients with confirmed GORD and 124 patients with Barrett's oesophagus with adequate gastric biopsies of whom 61 and 100 patients, respectively, were found to be $H$ pylori negative. Gastric biopsies taken from 50 patients presenting with dyspepsia and having (a) negative tests (biopsy urease and histology) for $H$ pylori infection, (b) a clinical history suggestive of non-ulcer dyspepsia (NUD) (that is, predominant epigastric pain, and no heartburn or reflux), and (c) no endoscopic evidence of peptic ulceration or Barrett's oesophagus, were used as controls. The archived slides of the gastric biopsies stained by haematoxylin-eosin and alcian blue/periodic acid Schiff were assessed (by MFD) "blind" to the clinical group. Foveolar hyperplasia, lamina propria oedema, vascular congestion, acute and chronic inflammation, and intestinal metaplasia in antral mucosa were graded on a $0-3$ scale (absent, mild, moderate, or marked) and the RGS and BRI were calculated for each case (see fig 1). While it was appreciated that NSAIDs and other factors affect RGS, and 
Table 1 Histological reflux gastritis scores (RGS) and bile reflux indices (BRI) based on assessment of antral biopsies from patients with non-ulcer dyspepsia (NUD), uncomplicated gastro-oesophageal reflux disease (GORD), and Barrett's oesophagus

\begin{tabular}{lcccl}
\hline Clinical group & Median & Range & Mean & $95 \%$ CI \\
\hline NUD (n=50) & & & & \\
$\quad$ RGS & 8 & $5-12$ & 8.4 & $7.8-9.0$ \\
$\quad$ BRI & 7 & $0-18$ & 7.8 & $6.3-9.3$ \\
GORD (n=61) & & & & \\
$\quad$ RGS & 8 & $5-12$ & 8.5 & $7.9-9.0$ \\
$\quad$ BRI & 11 & $0-24$ & 10.0 & $8.5-11.5$ \\
Barrett's (n=100) & & & & \\
$\quad$ RGS & 9 & $5-13$ & 8.9 & $8.5-9.2$ \\
$\quad$ BRI & 11 & $0-27$ & 12.5 & $11.2-13.8$ \\
\hline
\end{tabular}

possibly BRI, it was not possible in a retrospective study to ascertain an accurate drug or alcohol history.

REPRODUCIBILITY

To test inter- and intraobserver agreement, 50 cases selected at random from the overall series were graded for a second time by MFD "blind" to the previous results and by a second observer (NPM). RGSs and BRIs were calculated for each observer and used to test reproducibility.

\section{STATISTICAL ANALYSIS}

Summary descriptive statistics of the RGS and BRI results in each group were calculated. Overall differences between the three clinical groups were examined by one way analysis of variance for normally distributed data and the Kruskal-Wallis test for non-normal distributions. BRIs above and below 14 and RGSs above and below 10 in the three groups were compared using the $\chi^{2}$ test. Age, sex, foveolar hyperplasia score, vascular congestion score, and clinical group were entered into a stepwise multiple regression model to evaluate factors that independently predicted the BRI. The tests were carried out using SPSS version 9.0. Significance was taken at the 5\% level. With regard to the reproducibility of the histological assessments, we used kappa statistics to test for chance corrected agreement. This approach was developed for categorical data and therefore the BRI results were divided into low

Table 2 Frequency of high reflux gastritis scores $(R G S>10)$ and high bile reflux indices $(B R I>14)$ in the three clinical groups (patients with non-ulcer dyspepsia (NUD), uncomplicated gastro-oesophageal reflux disease (GORD), and Barrett's oesophagus)

\begin{tabular}{|c|c|c|c|c|c|c|}
\hline Clinical group & $n$ & \multicolumn{2}{|c|}{$R G S \leqslant 10$} & $R G S>10(\%)$ & $B R I \leqslant 14$ & $B R I>14(\%)$ \\
\hline NUD & 50 & \multicolumn{2}{|c|}{40} & $10(25 \%)$ & 46 & $4(8 \%)$ \\
\hline GORD & 61 & \multicolumn{2}{|c|}{49} & $12(20 \%)$ & 52 & $9(14.8 \%)$ \\
\hline Barrett's & 100 & \multicolumn{2}{|c|}{79} & $21(21 \%)$ & 71 & $29(29 \%)$ \\
\hline \multicolumn{7}{|c|}{$\begin{array}{l}\text { There was no significant difference in the distribution of RGSs between groups }\left(\chi^{2}=0.05 ; \text { NS }\right) \text { bu } \\
\text { the distribution of BRIs was highly significantly different }\left(\chi^{2}=10.65 ; p<0.005\right) .\end{array}$} \\
\hline \multicolumn{7}{|c|}{$\begin{array}{l}\text { Table } 3 \text { Histological bile reflux indices according to the clinicopathological subgroups } \\
\text { (patients with uncomplicated gastro-oesophageal reflux disease (GORD) and Barrett's } \\
\text { oesophagus) }\end{array}$} \\
\hline & \multicolumn{2}{|c|}{$n$} & Median & Range & Mean & $95 \% C I$ \\
\hline \multicolumn{7}{|l|}{ GORD } \\
\hline Mild/moderate & & 41 & 11 & $0-24$ & 10.1 & $8.0-12.1$ \\
\hline Severe & & 20 & 11 & $4-18$ & 10.0 & $8.0-12.0$ \\
\hline \multicolumn{7}{|l|}{ Barrett's } \\
\hline Short segment ${ }^{\star}$ & & 42 & 11 & $0-25$ & 12.1 & $10.4-14.4$ \\
\hline Long segment & & 58 & 12.5 & $0-27$ & 12.8 & $11.1-14.5$ \\
\hline
\end{tabular}

$\star$ Short segment, $<3 \mathrm{~cm}$ of columnar mucosa in the tubular oesophagus.
(0-7), medium (8-14), and high (>14) while RGSs were allocated to low (0-7), medium $(8-10)$, and high $(>10)$ categories. The results were analysed using the program "Agree" developed by Svanholm and colleagues. ${ }^{14}$

\section{Results}

As anticipated, the patient groups exhibited an increase in mean age with increasing oesophageal pathology. The NUD group had a mean age of 45.8 years (median 45.5; range 19-66), GORD patients had a mean age of 54.9 years (53; 28-87) while the Barrett's group had a mean age of 63.3 years $(65 ; 29-91)$.

The summary statistics for the RGSs and BRIs found in the three groups of patients are detailed in table 1 . The distribution of RGSs did not differ between groups but there was a highly significant difference in the distribution of BRIs (analysis of variance, $\mathrm{p}<0.0001$ ). When BRIs for the separate groups were compared, those in the Barrett's group were higher than those in the reflux oesophagitis (GORD) group which in turn were higher than those in the NUD patients. Likewise, when the distribution of patients with a BRI value above and below 14 were compared (table 2), there were highly significant differences between the three groups $\left(\chi^{2}=11, \mathrm{df}=2, \mathrm{p}<0.005\right)$. There was no difference in the distribution of high RGSs $(>10)$ between groups $\left(\chi^{2}=0.05, \quad \mathrm{df}=2\right.$, $\mathrm{p}=0.98)$. When the BRIs within the clinical subgroups were compared (table 3 ), there was no significant difference between GORD patients with either mild or moderate reflux and those with severe reflux, or between Barrett's patients with short or long segment involvement.

Patients with a BRI $>14$ were significantly older than patients with a BRI $\leqslant 14$ (mean age difference 8.6 ; 95\% confidence intervals 3.913.3 years). The Barrett's group were also significantly older than the other groups and it is possible that age could be a confounding factor in the association between BRI and Barrett's oesophagus. To explore this we entered age, sex, foveolar hyperplasia score, vascular congestion score, and clinical group into a linear multiple regression model with BRI as the dependent variable. Clinical group remained independently associated with bile reflux with a 1.4 (95\% confidence intervals $0.5-2.3$ ) unit increase in BRI in the GORD group compared with the NUD group and in Barrett's group compared with the GORD group $(p=0.004)$.

With regard to reproducibility of histological grading, interobserver agreement on the three levels of BRI was poor (38\%), with a kappa value of only 0.051 . This was largely a consequence of haphazard disagreement $\mathrm{t}^{15}$ on the grade of lamina propria oedema which is a much more subjective feature than chronic inflammation and intestinal metaplasia. Because of the high weighting given to this feature, a one grade difference in oedema translated into a 7 point difference in BRI. When the agreement on two categories of BRI, "low/medium" versus "high" (>14) was examined, overall agreement was improved $(78 \%)$ but because of high chance agreement the 
kappa value was only "fair" at 0.297. The agreement between observers on the three levels of reflux gastritis scores was "fair" (agreement $62 \%$; kappa 0.261 ) and when converted into the dichotomous grouping of "low/ moderate" versus "high", agreement rose to $74 \%$ but kappa was virtually unchanged (0.276). As would be expected, intraobserver agreement on the grade (low/medium/high) of BRI was improved at $66 \%$ and the kappa value (0.461) was "moderately good". Agreement on "low/medium" versus "high" reached $84 \%$ but without any substantial improvement in kappa (0.561).

\section{Discussion}

While the relationship between antral histology and duodeno-gastric reflux has been the subject of numerous studies in the past, these are of strictly limited value because they did not take into account $H$ pylori infection. Most recent studies ${ }^{916-18}$ have generally demonstrated a significant correlation between the histological picture of "reflux gastritis" and the degree of duodeno-gastric reflux or intragastric bile levels, but when Stein and colleagues ${ }^{19}$ investigated the diagnostic value of histology, they found it to be a poor predictor of excessive reflux with a positive predictive value of less than $60 \%$. However, foveolar hyperplasia has invariably been used in these studies, either alone or as part of a reflux score, yet we found that this feature was not a significant independent indicator of intragastric bile and therefore did not enter into the BRI. ${ }^{10}$

To our knowledge there have been only two previous studies which aimed to relate "reflux changes" in antral histology to oesophageal reflux diseases: one employed the "reflux gastritis score" and the other used the BRI. In the former, Haber and Lopez ${ }^{20}$ found a low prevalence of raised reflux scores in subjects with GORD but did not examine Barrett's patients, while Mason and Bremner ${ }^{21}$ used the BRI to compare groups of Barrett's patients with and without complications (stricture, ulcer, and carcinoma) and found no significant difference. However, they did not study patients with GORD or NUD. In the present study, we have shown that the RGSs did not differ between the three clinical groups. However, having previously established that the histological BRI is a better indicator of the degree of intragastric bile reflux, particularly in $H$ pylori negative subjects, we have demonstrated significantly higher scores in patients with Barrett's oesophagus than those with uncomplicated GORD or NUD. Patients with short or long segment Barrett's did not differ in their bile reflux indices. Using this index as a surrogate for intragastric bile concentrations it seems likely that Barrett's patients will have more bile (and therefore greater concentrations of other duodenal juice constituents) in the intraoesophageal refluxate than these other subjects. Such an outcome is not unexpected; there is much recent evidence pointing to a role for DGOR, and particularly for bile acids, in the pathogenesis of Barrett's oesophagus. ${ }^{22-25}$
A combination of acid and bile reflux offers a credible explanation for the histological components of Barrett's oesophagus. Sustained acid injury to the lower oesophagus would be expected to lead to gastric metaplasia. Gastric metaplasia is apparent as upward extension of the cardia zone and is an acknowledged feature of GORD. ${ }^{26}$ Indeed, it may be a general phenomenon that antedates the development of so-called "specialised" Barrett's metaplasia. ${ }^{27}$ The latter consists of goblet cells and intervening mucus rich vacuolated cells which secrete variable amounts of sulphomucin $^{28}$ and express a mixture of gastric and intestinal mucin genes. ${ }^{29}$ These morphological and histochemical characteristics and the pattern of mucin gene expression are identical to the incomplete type intestinal metaplasia (type III) found in gastric mucosa. Our previous demonstration of a relationship between intragastric bile reflux and type III intestinal metaplasia in gastric mucosa, ${ }^{10}$ mainly in the post-surgical stomach, is therefore entirely analogous to a role for acid and bile in the pathogenesis of Barrett's metaplasia. The absence of type III metaplasia from the antrum of many subjects with Barrett's oesophagus may be explicable in terms of changes in $\mathrm{pH}$ and the character of the refluxate after passage through the intact stomach.

The increased risk of adenocarcinoma in Barrett's oesophagus is also understandable in terms of progression from a "specialised" metaplasia equivalent to type III intestinal metaplasia under the influence of bile reflux. Type III (incomplete) intestinal metaplasia, but not type I (complete metaplasia), in the stomach carries a much greater risk of gastric cancer development. ${ }^{30}{ }^{31}$ Furthermore, bile acids, particularly in an alkaline milieu, could be the source of carcinogens which act on the metaplastic mucosa to produce neoplasia. ${ }^{32}$ While an association between Barrett's oesophagus and bile reflux (as indicated by this and other studies) does not necessarily point to causality, it is certainly plausible that the presence of bile derivatives and metaplasia in the oesophagus equivalent to type III intestinal metaplasia are linked to cancer development.

This conclusion has potentially important implications for the management of patients with intestinal metaplasia at the cardia. There are clear differences in demographic features and disease associations between subjects with complete and incomplete intestinal metaplasia at the cardia. ${ }^{33}$ Extrapolating from our knowledge of metaplasia in the stomach, one can speculate that complete intestinal metaplasia at the cardia has little or no malignant potential while type III intestinal metaplasia is likely to be part of Barrett's spectrum and will carry an increased risk of adenocarcinoma. ${ }^{35}$

If a direct role for refluxed bile derivatives in carcinogenesis becomes accepted, therapy aimed principally at acid reduction cannot be expected to eliminate the cancer risk in Barrett's oesophagus. It is true that proton pump inhibitors diminish bile reflux into the oesophagus, ${ }^{86}$ possibly by reducing overall 
gastric juice volume, but the concomitant generation of unconjugated bile acids in a relatively alkaline milieu may exacerbate oesophageal mucosal damage. ${ }^{37}$ The most effective and physiological way to diminish DGOR is by antireflux surgery. ${ }^{38} 39$

One conclusion from our study could be that those subjects with GORD who have clear evidence of bile related damage to the antral mucosa - that is, those with a high BRI-may be at increased risk of Barrett's oesophagus. Equally, one could predict that patients with Barrett's oesophagus and a high antral BRI are at increased risk of dysplasia and adenocarcinoma. However, there are important caveats to such speculation. The association between BRI and Barrett's oesophagus may not be causal and could be explained by other confounding factors not evaluated in this study. The BRI was derived from previous observations of one observer (MFD) but was poorly reproduced by an experienced second observer. Thus while we believe that the finding of significant differences in bile related changes in antral histology between NUD, GORD, and Barrett's subjects is valid in the particular context of a bile reflux index based on the same observer's experience, our findings require confirmation by comparing histological grading to direct measurements of intragastric bile reflux in similar clinical groups.

We are grateful to Knoll Ltd for an unrestricted grant in support of Dr Neville during the conduct of this study. Thanks are due to Tim Branch for pathology computer searches and to Les Beck for slide retrieval. Paul Moayyedi is funded by a UK MRC Health Services Research Training Fellowship.

1 Slack JMW. Epithelial metaplasia and the second anatomy.

Lancet 1986;ii: $268-71$.
2 Slack JMW. Stem cells in epithelial tissues. Science 2000;287:1431-3.

3 Wyatt JI, Rathbone BJ, Dixon MF, et al. Campylobacter Wyatt JI, Rathbone BJ, Dixon MF, et al. Campylobacter
pyloridis and acid induced gastric metaplasia in the pathopyloridis and acid induced gastric metaplasia in the
genesis of duodenitis. $\mathcal{F}$ Clin Pathol 1987;40:841-8.

4 Craanen ME, Dekker W, Blok P, et al. Intestinal metaplasia Craanen ME, Dekker W, Blok P, et al. Intestinal metaplasia
and Helicobacter pylori: an endoscopic bioptic study of the and Helicobacter pylori: an endoscopic

5 Eidt S, Stolte M. Antral intestinal metaplasia in Helicobacter pylori gastritis. Digestion 1994;55:13-18

6 Nasmyth DG, Godwin PG, Dixon MF, et al. Ileal ecology after pouch-anal anastomosis or ileostomy. A study of mucosal morphology, fecal bacteriology, fecal volatile fatty acids, and their interrelationship. Gastroenterology 1989;96: 817-24.

7 Marshall EEK, Anggiansah A, Owen WJ. Bile in the oesophagus: clinical relevance and ambulatory detection. Br F Surg 1997;84:21-8.

8 Champion G, Richter JE, Vaezi MF, et al. Duodenogastroesophageal reflux: relationship to $\mathrm{pH}$ and importance in esophageal reflux: relationship to $\mathrm{pH}$ and importance

9 Dixon MF, O'Connor HJ, Axon ATR, et al. Reflux gastritis: distinct histopathological entity? f Clin Pathol 1986;39: 524-30.

10 Sobala GM, O'Connor HJ, Dewar EP, et al. Bile reflux and intestinal metaplasia in gastric mucosa. F Clin Pathol 1993; 46:235-40.

11 Sobala GM, King RFJG, Axon AT, et al. Reflux gastritis in the intact stomach. F Clin Pathol 1990;43:303-6.

12 Moayyedi P, Wason C, Peacock R, et al. Changing patterns of gastritis in long-standing acid suppression: Effect of
Helicobacter pylori eradication. Helicobacter 2000;5:206-14.
13 O'Connor HJ, Wyatt JI, Dixon MF, et al. Campylobacterlike organisms and reflux gastritis. F Clin Pathol 1986;39: like organ.

14 Svanholm H, Starklint H, Gundersen HJG, et al. Reproducibility of histomorphologic diagnoses with special reference to the kappa statistic. APMIS 1989;97:689-98.

15 Silcocks PBS. Measuring repeatability and validity of histoogical diagnosis - a brief review with some practical examples. 7 Clin Pathol 1983;36:1269-75.

16 Bechi P, Amorosi A, Mazzanti R, et al. Gastric histology and fasting bile reflux after partial gastrectomy. Gastroenterology 1987;93:335-43.

17 Offerhaus GJ, Rieu PN, Jansen JB, et al. Prospective comparative study of the influence of postoperative bile reflux on gastric mucosal histology and Campylobacter reflux on gastric mucosal histology
pylori infection. Gut 1989;30:1552-7.

18 Bechi P, Amorosi A, Mazzanti R, et al. Reflux-related gastric mucosal injury is associated with increased mucosal histamine content in humans. Gastroenterology 1993;104:105763

19 Stein Hj, Smyrk TC, DeMeester TR, et al. Clinical value of endoscopy and histology in the diagnosis of duodenogastric reflux disease. Surgery 1992;112:796-804.

20 Haber MM, Lopez I. Reflux gastritis in gastroesophageal reflux disease: A histopathological study. Ann Diagn Pathol 1999;3:281-6.

21 Mason RJ, Bremner CG. Gastritis in Barrett's esophagus. World f Surg 1995;19:96-101.

22 Vaezi MF, Richter JL. Role of acid and duodenogastroesophageal reflux in gastroesophageal reflux disease. Gastroenterology 1996;111:1192-9.

23 Marshall REK, Anggiansah A, Owen WA, et al. The temporal relationship between oesophageal bile reflux and $\mathrm{pH}$ in gastro-oesophageal reflux disease. Eur $\mathcal{F}$ Gastroenterol Hepatol 1998;10:385-92.

24 Stein HJ, Kauer WK, Feussner H, et al. Bile reflux in benign and malignant Barrett's esophagus: effect of medical acid suppression and Nissen fundoplication. $f$ Gastrointest Surg 1998;2:333-41.

25 Nehra D, Howell P, Williams CP, et al. Toxic bile acids in gastro-oesophageal reflux disease: influence of gastric acidity. Gut 1999;44:598-602.

26 Chandrasoma PT, Lokuhetty DM, Demeester TR, et al. Definition of histopathologic changes in gastroesophageal reflux disease. Am $\mathcal{F}$ Surg Pathol 2000;24:344-51.

27 DeMeester SR, DeMeester TR. Columnar mucosa and intestinal metaplasia of the oesophagus: fifty years of controversy. Ann Surg 2000;231:303-21.

28 Rothery GA, Patterson JE, Stoddard CJ, et al. Histological and histochemical changes in the columnar lined (Barrett's) oesophagus. Gut 1986;27:1062-8.

29 Arul GS, Moorghen M, Myerscough N, et al. Mucin gene expression in Barrett's oesophagus: an in situ hybridisation and immunohistochemical study. Gut 2000;47:753-61.

30 Filipe MI, Muñoz N, Matko I, et al. Intestinal metaplasia types and the risk of gastric cancer: a cohort study in Slovenia. Int f Cancer 1994;57:324-9.

31 Wu M-S, Shun C-T, Lee W-C, et al. Gastric cancer risk in relation to Helicobacter pylori infection and subtypes of intestinal metaplasia. Br f Cancer Res 1998;78:125-8.

32 Busby WF, Shuker DEG, Charnley G, et al. Carcinogenicity in rats of the nitrosated bile acid conjugates $\mathrm{N}$-nitrosoglycocholic acid and $\mathrm{N}$-nitrosotaurocholic acid. Cancer Res 1985;45:1367-71.

33 Guenther T, Hackelsberger A, Roessner A, et al. Subtyping of intestinal metaplasia at the gastroesophageal junctionuseful for differentiation of reflux and H. pylori. Gastroenterology 1999;116:A178.

34 Voutilainen M, Farkkila M, Juhola M, et al. Complete and incomplete intestinal metaplasia at the oesophagogastric unction: prevalences and associations with endoscopic erosive oesophagitis and gastritis. Gut 1999;45:644-8.

35 Bowrey DJ, Clark GWB, Williams GT. Patterns of gastritis in patients with gastro-oesophageal reflux disease. Gut 1999;45:798-803.

36 Marshall RE, Anggiansah A, Manifold DK, et al. Effect of omeprazole $20 \mathrm{mg}$ twice daily on duodenogastric and gastro-oesophageal bile reflux in Barrett's oesophagus. Gut 1998;43:603-6.

37 Theisen J, Nehra D, Citron D, et al. Suppression of gastric acid secretion in patients with gastroesophageal reflux disease results in gastric bacterial overgrowth and deconjugation of bile acids. F Gastrointest Surg 2000;4:50-4

38 Triadafilopoulos G. Proton pump inhibitors for Barrett's oesophagus. Gut 2000;46:144-6.

39 DeMeester TR. Anti-reflux surgery in the management of Barrett's esophagus. F Gastrointest Surg 2000;4:124-8. 